\title{
Measurement of Drell-Yan and associated jet cross section at low and high invariant masses
}

\author{
Samantha Katherine DOOLING* \\ On behalf of the CMS Collaboration \\ ( Deutsches Elektronen-Synchrotron ( DESY)) \\ E-mail: samantha.dooling@desy.de
}

\begin{abstract}
Measurements of the differential Drell-Yan and associated jet cross section as a function of the Drell-Yan mass are presented using an integrated luminosity of $4.9 \mathrm{fb}^{-1}$ in the di-muon channel of proton-proton collision data recorded with the CMS detector at the LHC at $\sqrt{s}=7 \mathrm{TeV}$. Cross sections as a function of the Drell-Yan transverse momentum are measured differentially in the Drell-Yan mass range of 30 to $1500 \mathrm{GeV}$. The $p_{\mathrm{T}}$ spectrum of the Drell-Yan allows to study multiple-gluon emissions and resummation effects. The cross section for Drell-Yan production in association with one or two jets with $p_{\mathrm{T}}>30 \mathrm{GeV}$ in the range $|\eta|<4.5$ are measured. Multi-jet emissions in a rapidity interval between the Drell-Yan and the leading jet is a sensitive probe for multi-gluon emissions. The jet multiplicity in Drell-Yan production as a function of the rapidity separation of the leading jet and the Drell-Yan is presented. All measurements are compared to Monte Carlo predictions including matrix elements plus parton showers.
\end{abstract}

XXII. International Workshop on Deep-Inelastic Scattering and Related Subjects, 28 April - 2 May 2014

Warsaw, Poland

* Speaker. 


\section{Introduction}

The production of Drell-Yan (DY) lepton pairs in hadron-hadron collisions is a suitable process to study the effect of the soft-gluon resummation in perturbative quantum chromodynamics (pQCD). While DY lepton pair production at large transverse momenta can be described by fixed order calculations in $\mathrm{pQCD}$, at small $p_{\mathrm{T}}$ resummation of soft gluons to all orders in perturbation theory is required $[1,2]$.

Thus, the $p_{\mathrm{T}}$ spectrum of the DY lepton pair is of special interest to test contribution from nonperturbative and perturbative multi-gluon resummation. In the case of the inclusive DY production the maximum of the DY $p_{\mathrm{T}}$ distribution is around $5 \mathrm{GeV}$. When requiring DY in association with additional jets the maximum shifts to higher $p_{\mathrm{T}}$ and the phase space for multi-gluon emissions is enlarged. In Fig. 1 the comparison of inclusive DY production to DY and at least one jet above a $p_{\mathrm{T}}$ of $30 \mathrm{GeV}$ is shown. When switching of the effect of parton shower (PS) in the Monte Carlo (MC) generation, the cross section in $p_{\mathrm{T}}$ drops to zero just below the peak. Thus, the rise at small $p_{\mathrm{T}}$ is simulated by the PS algorithm in the MC event generator.

The detailed measurements of inclusive DY lepton pair production as well as DY lepton pair production in association with jets, especially in the mass range above the $Z$ mass (and in the range of $m^{\mu \mu} \sim 125 \mathrm{GeV}$ ), is important for a later comparison with Higgs production, which can be used to determine the differences of soft gluon and multi-jet resummation in a quark or gluon induced process [3].

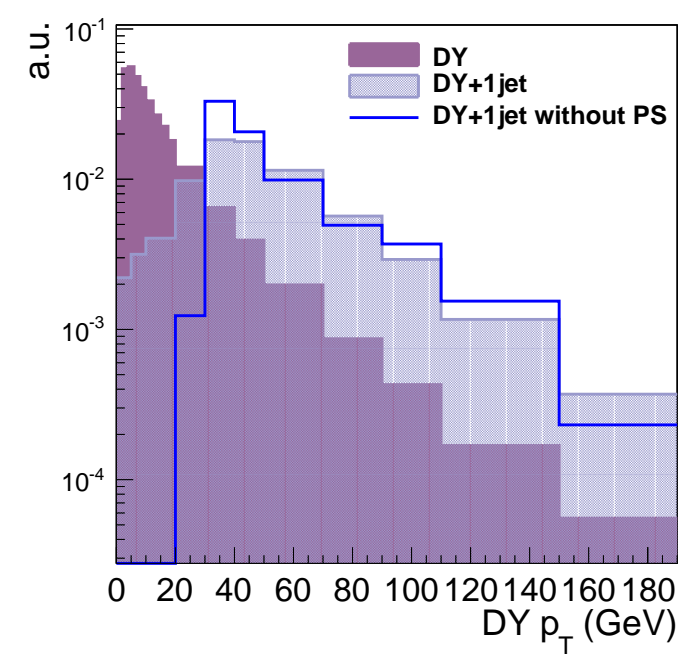

Figure 1: Effect of the parton shower in the Drell-Yan $p_{\mathrm{T}}$ distribution

Here a measurement of the differential DY and associated jet cross section for various ranges of DY di-muon mass $m^{\mu \mu}$ using an integrated luminosity of $4.9 \mathrm{fb}^{-1}$ in the di-muon channel of protonproton collision data recorded with the CMS detector at the LHC at $\sqrt{s}=7 \mathrm{TeV}$ is presented [4]. Differential cross sections as a function of the DY di-muon transverse momentum are measured differentially in the di-muon mass range of $30<m^{\mu \mu}<1500 \mathrm{GeV}$. The cross sections for DY 
production in association with at least one or two jets (in the following DY +1 jet and DY +2 jets, respectively) with $p_{\mathrm{T}}^{\text {jet }}>30 \mathrm{GeV}$ in the range $\left|\eta^{\text {jet }}\right|<4.5$ are measured, where $\eta=-\ln [\tan (\theta / 2)]$ and $\theta$ is the polar angle with respect to the anti-clockwise beam direction. Jets are reconstructed by the anti- $k_{\mathrm{T}}$ algorithm with a radius parameter of 0.5 .

\section{Transverse Momentum Distribution}

The measurement is performed for a pair of muons with $\left|\eta^{\mu}\right|<2.1$ and $p_{\mathrm{T}}^{\text {lead } \mu}>20 \mathrm{GeV}$ and $p_{\mathrm{T}}^{\text {sublead } \mu}>10 \mathrm{GeV}$ and a DY di-muon mass $30<m^{\mu \mu}<1500 \mathrm{GeV}$. Jets are required to have $p_{\mathrm{T}}^{\text {jet }}>30 \mathrm{GeV}$ with $\left|\eta^{\text {jet }}\right|<4.5$. The data points are corrected for detector effects to stable-particle level and are compared to different Monte Carlo predictions. The cross section in the $Z$ peak region $\left(60<m^{\mu \mu}<120 \mathrm{GeV}\right)$ integrated over the di-muon $p_{\mathrm{T}}, \sigma_{\mathrm{Z}}$, is used to normalize all cross sections for the three different topologies (inclusive, DY+1 jet, DY+2 jets) to reduce systematic uncertainties. The main systematic uncertainties are due to Jet Energy Corrections and are around 10\%.

The double differential cross section for inclusive DY production is shown in fig. 2 for five different bins in the invariant mass $m^{\mu \mu}$. Overall a reasonable agreement between data and the predictions from MADGRAPH+PYTHIA6 is observed. With increasing invariant mass the perturbative high $p_{\mathrm{T}}$ tail flattens while the low $p_{\mathrm{T}}$ region is not sensitive to the di-muon invariant mass.

The di-muon $p_{\mathrm{T}}$ distribution for inclusive DY, DY +1 jet, and DY +2 jets production in the $Z$ invariant mass region $(60-120 \mathrm{GeV})$ is presented in fig. 3 . The corrected data points are compared to different matrix element plus parton shower predictions. The MC generators PYTHIA6, POWHEG+PYTHIA6, and MADGRAPH+PYTHIA6 are used. The parton shower is modelled in all cases by PYTHIA6, while the matrix element calculations of the DY process correspond to different calculations in orders in perturbation theory. The lowest order and first order calculation in $\alpha_{\mathrm{S}}$ is generated by PYTHIA. POWHEG produces a DY boson and two hard emissions plus the first hard emission from the parton shower, and MADGRAPH produces the DY boson with maximum four hard partons in the matrix element calculation. The comparison to data as shown in fig. 3 presents a good agreement of all MC generators in the inclusive DY production (left). For DY+1 jet the high $p_{\mathrm{T}}$ tail can be described well by all MC predictions while the low $p_{\mathrm{T}}$ region can only be described by the higher order calculations. In the case of the DY +2 jets the cross section at low $p_{\mathrm{T}}$ is increased. The DY is mostly balanced by the soft gluons from the parton shower algorithm. The higher order calculations from MADGRAPH provides the best agreement to data.

\section{Absolute Rapidity Separation}

Multi-jet emissions in a pseudorapidity interval between the DY lepton pair and a leading jet are a sensitive probe for multi-gluon emissions At large rapidity separation between the DY lepton pair and the jet, a fixed order approach is expected to fail, and again perturbative multi-jet resummation might play a significant role and investigations of angular distributions with a forward DY pair are sensitive to small-x resummation. Of additional interest is the jet multiplicity between the DY lepton pair and the leading jet, as resummation might lead to a different behaviour compared to a fixed order prediction. The differential cross section of the absolute rapidity separation $\left|\Delta y\left(\mathrm{DY}, j_{1}\right)\right|$ is presented for the production of DY +1 jet. The cross section is shown in the mass 

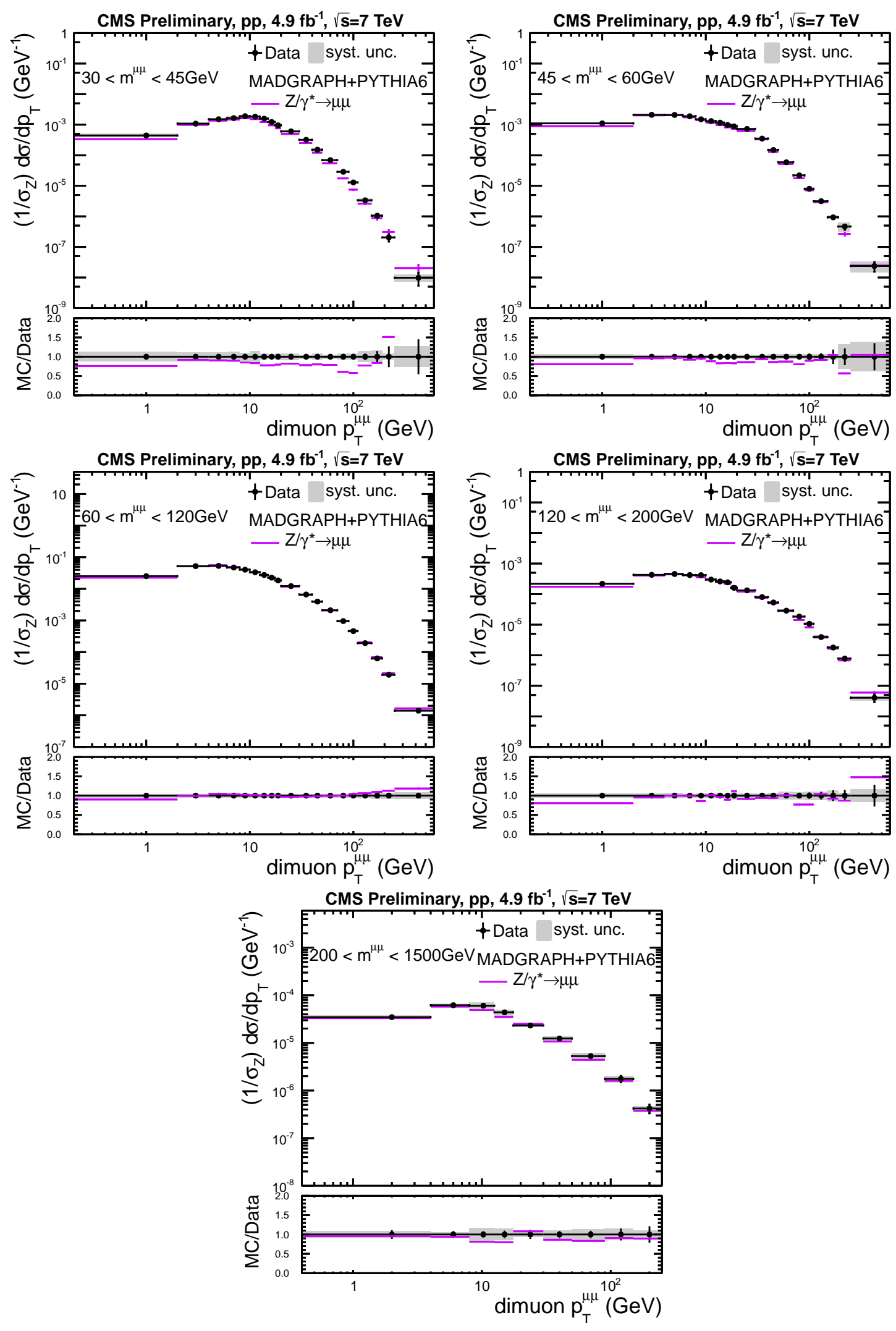

Figure 2: Inclusive di-muon $p_{\mathrm{T}}$ distribution for five different invariant mass bins. Corrected data is compared to MADGRAPH+PYTHIA6 MC. 

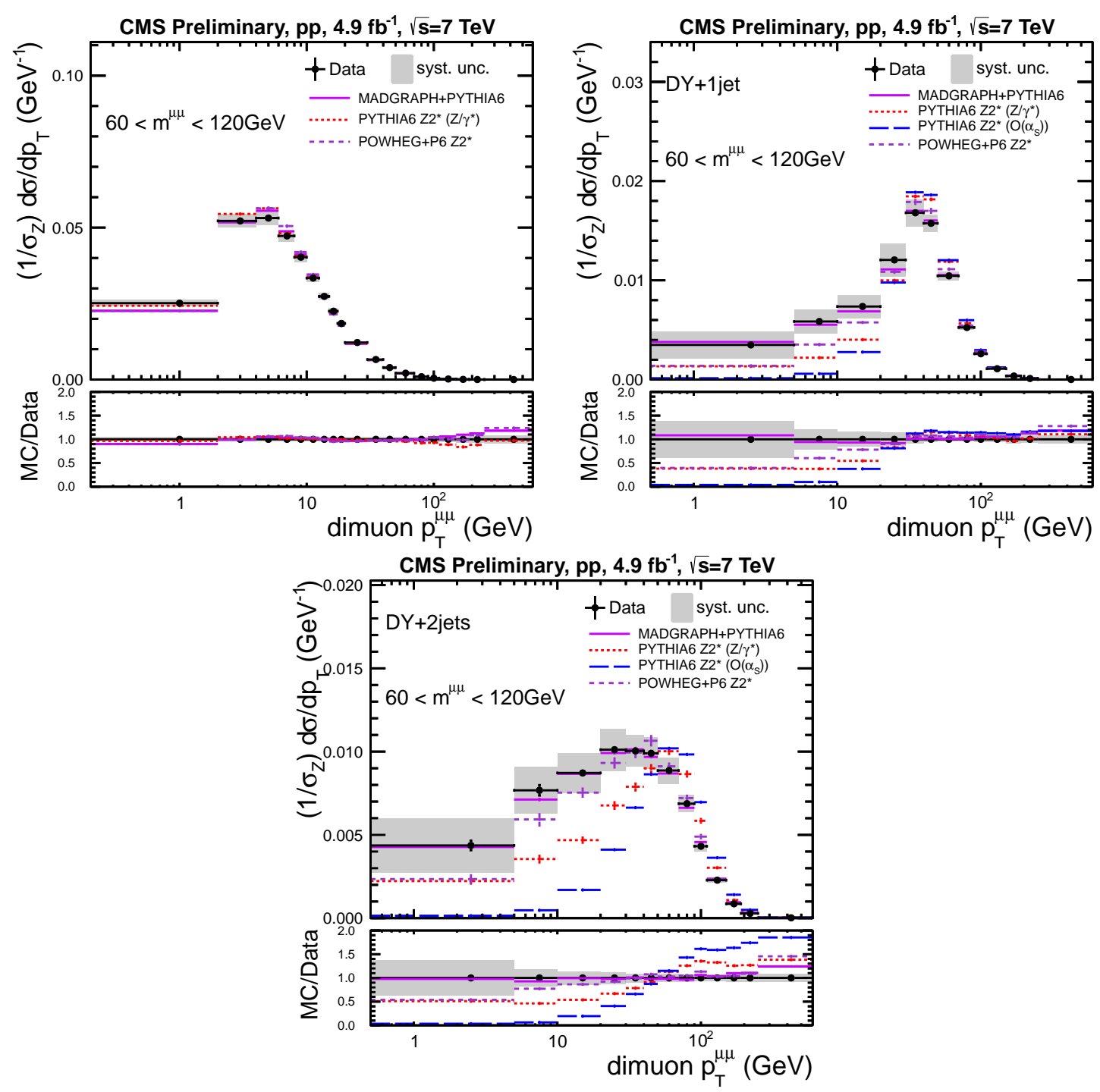

Figure 3: Di-muon $p_{\mathrm{T}}$ distribution in the $Z$ invarinat mass bin for inclusive DY, DY +1 jet, and DY +1 jets poduction. Corrected data is compared to different MC generators.

bin $60-120 \mathrm{GeV}$ in fig. 4 (left). The DY pair is required to fulfil $|\eta|>2.5$. All cross sections are normalized to the $\mathrm{Z}$ boson mass region $(60-120 \mathrm{GeV})$.

The cross section as a function of the rapidity separation shows the best agreement to the higher order perturbative calculations. The lower order calculation fail to describe data at large rapidity separation.

The average jet multiplicity of jets with $p_{\mathrm{T}}>30 \mathrm{GeV}$ between the leading jet and the forward DY production as a function of the rapidity separation $\Delta y(\mu \mu, \mathrm{j})$ is presented in Fig. (4) (right). The average jet multiplicity increases with increasing separation between the DY lepton pair and the leading jet from 0 to 0.3 at large rapidity separation. The general behaviour is reproduced by the MADGRAPH and POWHEG simulation. The lowest order predictions from PYTHIA are not able to reproduce the average jet multiplicity. 

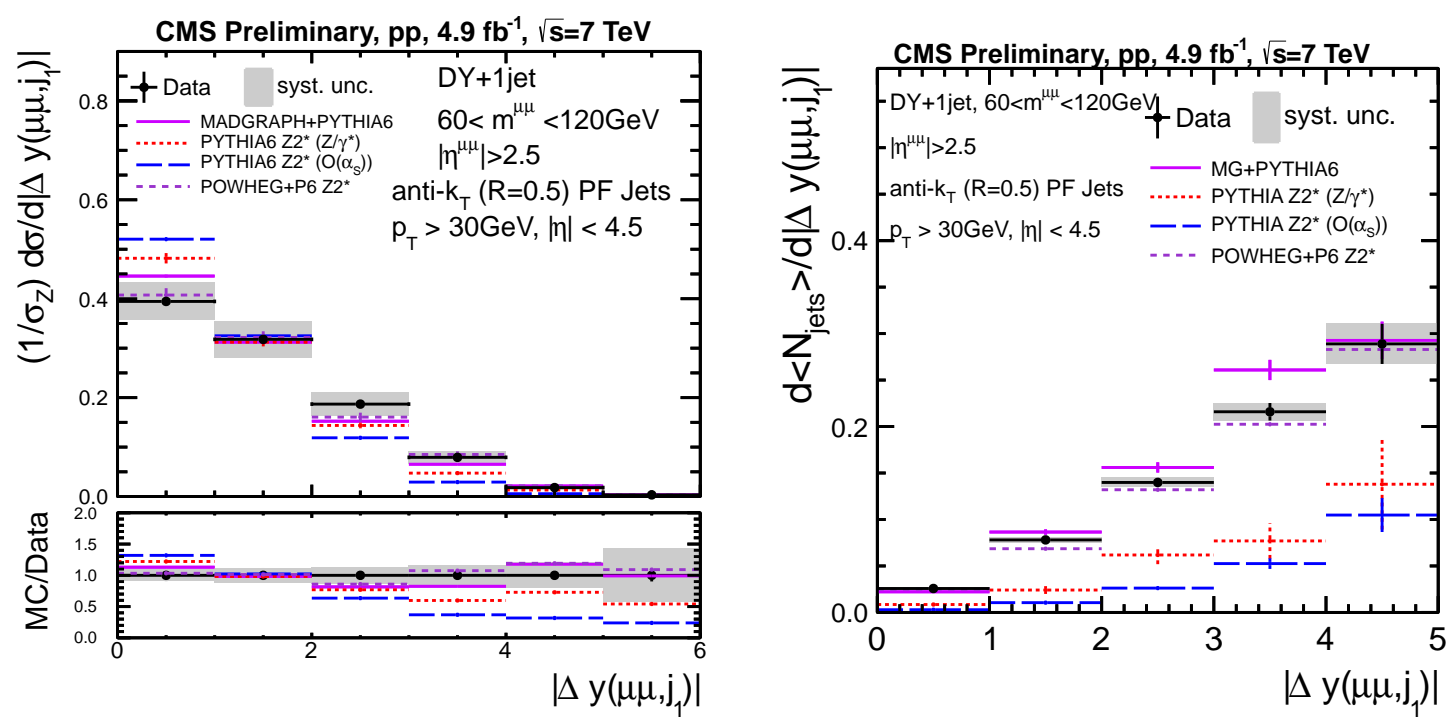

Figure 4: Cross section as a function of the absolute rapidity separation between the forward DY and the leading jet in DY+1jet (left) and the average jet multiplicity (right). Corrected data is compared to different MC predictions.

\section{Conclusions}

The first measurement of the differential DY and associated jets cross section as a function of the DY di-muon mass is presented. We use DY+jets to enlarge the phase space at low $p_{\mathrm{T}}$, which is sensitive to soft-gluon resummation. Corrected data is compared to matrix element plus parton shower calculations and the best agreement is obtained by higher order calculations perturbation calculations from MADGRAPH+PYTHIA. The lowest order calculations in $\alpha_{\mathrm{S}}$ fail to describe the low $p_{\mathrm{T}}$ region as well as the high rapidity separation. Concluding, when requiring additional jets in association with DY production the phase space for resummation can be enlarged but higher order calculations with matched partons showers are needed to describe the multi-jet emissions.

\section{Acknowledgements}

We are grateful to the DIS organizers and staff for the invitation to an exciting conference. We want to thank all DIS participants for a nice atmosphere and interesting discussions.

\section{References}

[1] Dokshitzer, Y. L. and Diakonov, D. and Troian, S. I., Phys. Lett. B79 (1978) 269.

[2] Collins, J. C. and Soper, D. E. and Sterman, G. F., Nucl. Phys. B250 (1985) 199.

[3] Cipriano, P. and Dooling, S. and Grebenyuk, A. and Gunnellini, P. and Hautmann, F. and Jung, H. and Katsas, P., Phys. Rev. D88 (2013) 097501.

[4] CMS Collaboration, CMS-PAS-FSQ-13-003, "Measurement of the double differential Drell-Yan and associated jet cross sections at low and high invariant masses in proton-proton collisions at $\sqrt{s}=7$ TeV", http://cds.cern.ch/record/1670353 (2014). 